\title{
A full complement of receptors in immune complex diseases
}

\author{
Jeffrey V. Ravetch \\ Laboratory of Molecular Genetics and Immunology, Rockefeller University, New York, New York, USA \\ J. Clin. Invest. 110:1759-1761 (2002). doi:10.1172/JCI200217349.
}

In this issue of the JCI, Shushakova et al. (1) describe experimental results that provide a direct link between the $\mathrm{C} 5 \mathrm{a}$ anaphylotoxin and the $\operatorname{IgG} \mathrm{Fc}$ receptors (Fc $\gamma \mathrm{Rs}$ ) in mediating immune complex-triggered (IC-triggered) inflammatory disease. Using a murine lung model of IC-induced inflammation, the authors demonstrate that $\mathrm{C} 5 \mathrm{a}$, acting through the $\mathrm{C} 5 \mathrm{a}$ receptor $(\mathrm{C} 5 \mathrm{aR})$, exacerbates inflammation in part by altering the ratio of activation to inhibitory $\mathrm{F} c \gamma \mathrm{R}$ expression on alveolar macrophages, enhancing the former and suppressing the latter, thereby optimizing the ability of alveolar macrophages to respond to ICs and trigger cytokine release and neutrophil chemotaxis. C5a now joins the ranks of other regulators of $\mathrm{F} c \gamma \mathrm{R}$ expression, such as IFN- $\gamma$ and intravenous immunoglobulin (IVIG), in modulating IC-induced inflammation by acting on the primary IC targets, the Fc $\gamma$ Rs. What makes this observation worthy of comment is the historical context of IC-mediated inflammation and the confusion that has surrounded the basic mechanism of this fundamental immunological reaction, the Arthus reaction.

\footnotetext{
Address correspondence to: Jeffrey V. Ravetch, Laboratory of Molecular Genetics and Immunology, Rockefeller University, 1230 York Avenue, Box 98, New York, New York, 10021, USA. Phone: (212) 327-7321; Fax: (212) 327-7318;

E-mail: ravetch@rockefeller.edu.

Conflict of interest: The author has declared that no conflict of interest exists.

Nonstandard abbreviations used: IgG Fc receptor $(\mathrm{Fc} \gamma \mathrm{R})$; immune complex (IC); C5a receptor $(\mathrm{C} 5 \mathrm{aR})$; intravenous

immunoglobulin (IVIG); immunoreceptor tyrosine-based activation motif (ITAM); tyrosine-based inhibitory motif (ITIM); glucose-6-phosphate-isomerase (GPI).
}

The Arthus reaction, 1903-1996 In 1903 Maurice Arthus published his observations on the induction of a localized inflammatory reaction at the site of repeated immunizations with a foreign antigen (2). The Arthus reaction, as it has become known, results from the deposition of ICs in specific anatomic sites and the subsequent activation of inflammatory responses to these complexes. This reaction has served as the basis for dissecting the cellular and molecular events that are triggered by IC deposition and serves as the basis for our understanding of the pathophysiology of IC-mediated diseases, such as lupus and rheumatoid arthritis. While the role of antibody-antigen complexes is clearly established in the inflammatory response, the triggers and

mediators downstream of ICs have been more difficult to identify with certainty. The detailed in vitro observations that ICs bind early components of complement and result in their activation, leading to the generation of $\mathrm{C} 3$ as a central protein in complement activation, led to the widely held model of the mechanism of IC-mediated inflammation. In this model, ICs directly lead to C3 activation followed by the formation of the late components of complement activation, notably $\mathrm{C} 5 \mathrm{a}$ and the membrane attack complex. ICs, acting through complement pathways, were thus deemed to be responsible for all the downstream effector responses, including edema, hemorrhage, neutrophil infiltration, and the release of pro-inflammatory mediators such as

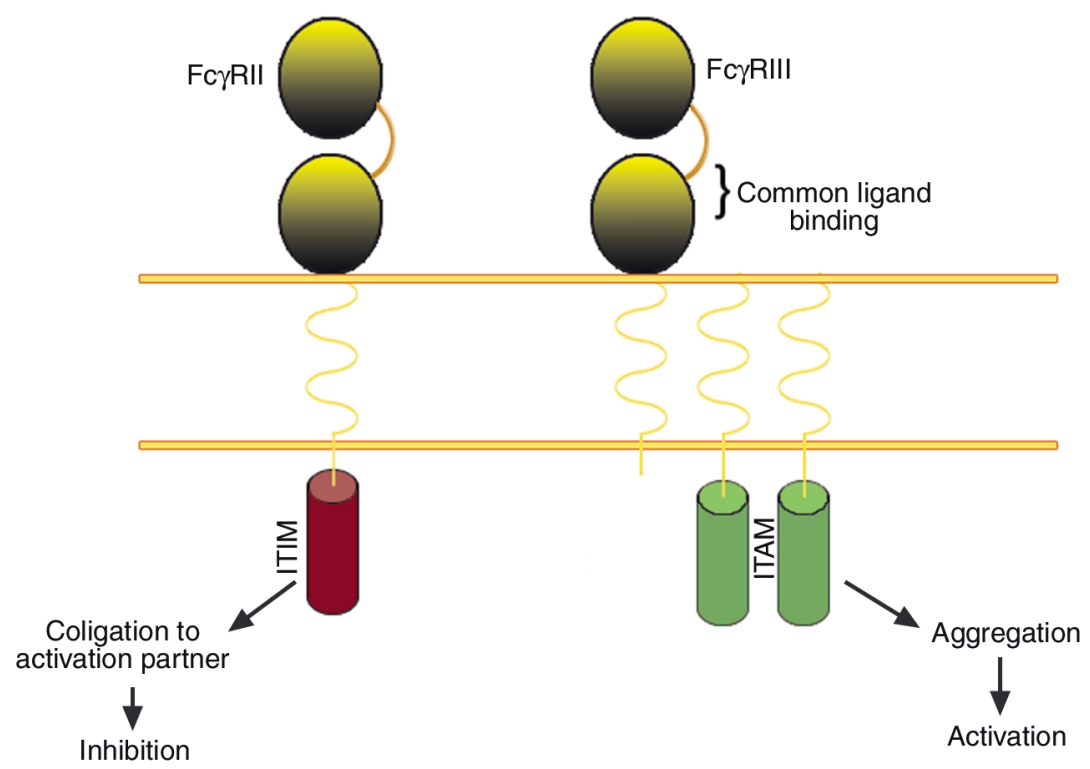

Figure 1

IgG Fc receptors are expressed as activation/inhibitory pairs. A schematic representation of an acti-

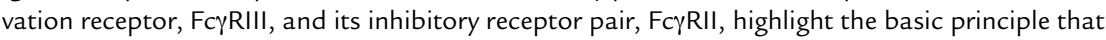
the interaction of two receptors with common ligand binding domains that mediate opposing signals will set a threshold for stimulation. 
TNF and IL-1 (3). The authors of these models did not contemplate the possibility of direct activation of effector cells by ICs, mediated by the cellular receptors for these complexes, the Fc $\gamma$ Rs (4). These cell surface receptors had been shown to be potent activators of effector cells upon their crosslinking by ICs. A unique aspect of the Fc $\gamma$ Rs was their heterogeneity, in which a conserved ligand-binding domain was associated with alternative intracytoplasmic signaling motifs (5) (Figure 1). These motifs could either activate cellular responses through the immunoreceptor tyrosine-based activation motif (ITAM) sequences or inhibit the activation response through immune receptor tyrosine-based inhibitory motif (ITIM) sequences. Activation and inhibitory FcyRs were found to be coexpressed on effector cells such as mast cells, macrophages, and neutrophils (6), thus leading to the hypothesis that the ratio of these opposing signaling receptors was critical in setting thresholds for the inflammatory activity of ICs (7).

\section{Mouse knockouts redefine the Arthus reaction}

Critical evaluation of the roles of these alternative pathways became possible with the advent of targeted gene disruption in the mouse in the 1990s. Disruption of activation FcyRs, notably Fc $\gamma$ RIII, resulted in ablation of ICmediated inflammatory responses, as well as the ability of cytotoxic antibodies to trigger in vivo clearance of opsonized cells or mediate antibodydependent cellular cytotoxicity in vivo (8). Conversely, disruption of the inhibitory FcyRIIB receptor led to enhancement of IC-mediated inflammation and antibody-triggered cytotoxicity and clearance of opsonized cells in vivo $(9,10)$. What of the role of complement then? Using the reverse passive Arthus reaction as a model for IC-triggered inflammation, researchers found that mice deficient in the classical, early components of complement, including the central activator $\mathrm{C} 3$, were able to mount a normal inflammatory response to ICs (11). C3-deficient mice, in general, where found to have a wildtype response to cytotoxic antibodytriggered inflammation and opsonized cell clearance $(8,12)$. In active models of IC deposition, such as anti-glucose- 6-phosphate-isomerase (GPI) arthritis, a partial dependence on C3 was observed, consistent with its role in stabilizing the IC (13). In contrast, loss of activation $\mathrm{Fc} \gamma \mathrm{Rs}$ ablated the inflammatory response in all models of IgGinduced inflammation, while loss of the inhibitory $\mathrm{Fc} \gamma \mathrm{R}$ enhanced the response. Further studies went on to demonstrate that it was the activation Fc $\gamma$ RIII receptor on mast cells that initiated the Arthus reaction, thus putting FcyRIII upstream of cytokine release and neutrophil influx $(14,15)$. In contrast, the late component $\mathrm{C} 5 \mathrm{a}$ was found to have a variable effect on IC-mediated inflammation in different experimental models, ranging from an essential role in the anti-GPI arthritis model to a modest one in IC alveolitis, suggesting that the specific details of each model, such as the strain background, could affect the outcome (15).

\section{Convergence of pathways}

How are we to understand the roles of Fc $\gamma$ Rs and complement in the ICinduced inflammatory response? The most consistent model that emerges from the genetic data of the last six years is the absolute requirement for

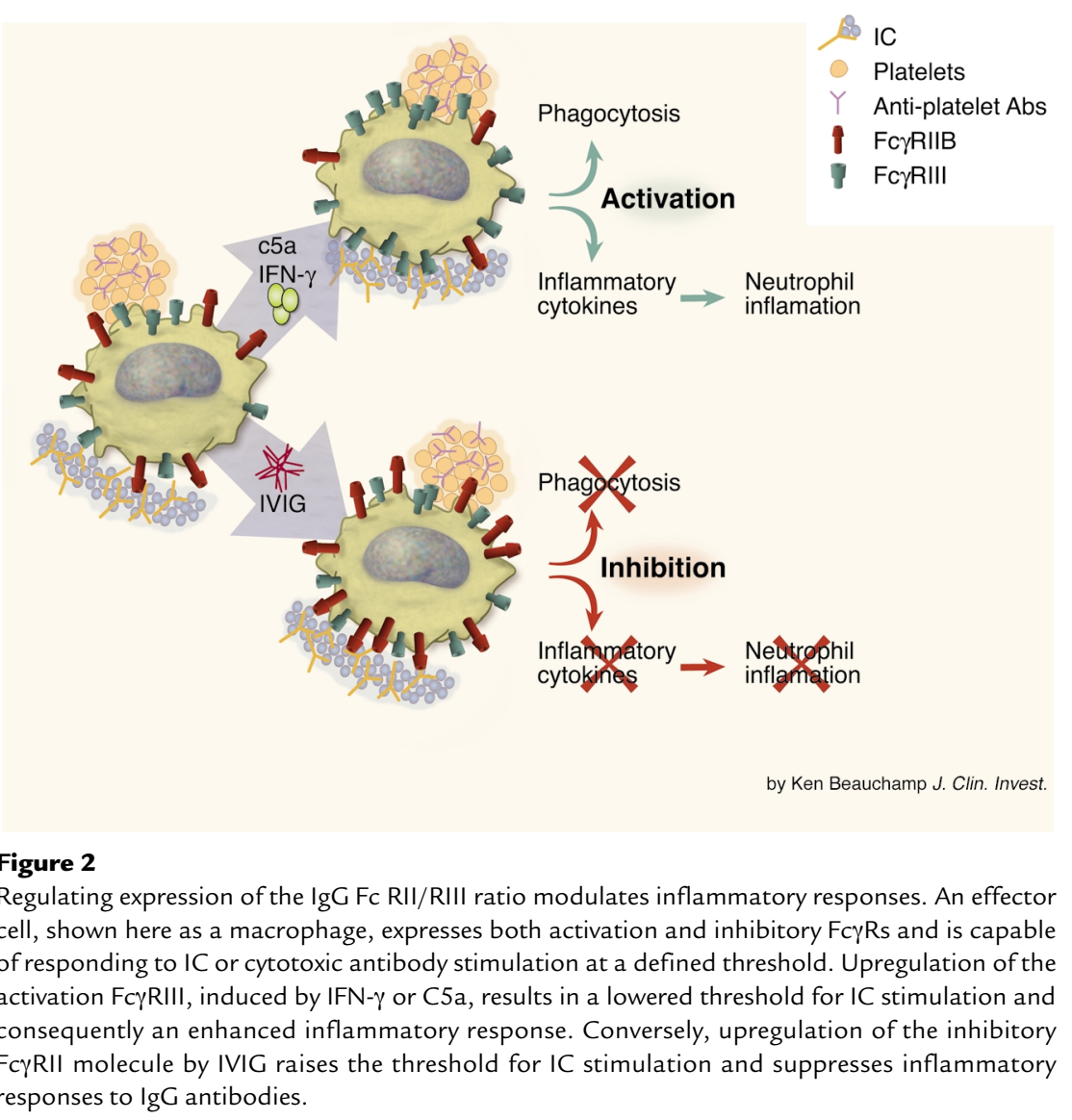

engagement of activation $\mathrm{Fc} \gamma \mathrm{Rs}$ by ICs. This activation is modulated by the expression ratio of activation and inhibitory Fc $\gamma$ Rs. Early complement components, including C3, are likely to be involved in IC clearance and stability and will thus play variable roles in induced models of IC-mediated inflammation. The late components, such as C5a, behave as downstream cytokines, activated by the triggering of Fc $\gamma$ Rs on effector cells such as mast cells. In this model, the essential step is the regulation of $F c \gamma R$ activation, and not complement. Fc $\gamma \mathrm{R}$ regulation, in turn, can be thought of in terms of the independent modulation of the activation and inhibitory receptors. This level of regulation has been observed in the developmental expression of these receptors on myeloid cells. Mast cells, macrophages, and dendritic cells, when they emerge from the bone marrow, primarily express the inhibitory Fc $\gamma$ RIIB, with little expression of the activation Fc $\gamma$ RIII, thus providing a simple mechanism to prevent inappropriate activation of these circulating cells by ICs (16). However, this ratio inverts in an inflammatory environment, where 
local production of cytokines such as IFN- $\gamma$ and TNF result in the upregulation of Fc $\gamma$ RIII and downregulation of Fc $\gamma$ RIIB (17). To this list of cytokines that regulate $\mathrm{Fc} \gamma \mathrm{R}$ expression we can now add C5a through $\mathrm{C} 5 \mathrm{aR}$, based on the results reported in this issue of the JCI by Shushakova and colleagues (1). The authors demonstrate that the ability of $\mathrm{C} 5 \mathrm{a}$ to upregulate Fc $\gamma$ RIII and downregulate Fc $\gamma$ RIIB accounts for its ability to augment IC-mediated inflammation and may also explain the variability in the data on the role of $\mathrm{C} 5 \mathrm{a}$ in various IC-induced models of inflammation. Fc $\gamma \mathrm{R}$ expression is under the redundant control of numerous other cytokines as well as genetic background effects; thus specific strains and models will likely have variable dependence on a specific cytokine pathway. Once maximal levels of the activation/inhibitory ratio are achieved, further augmentation is not likely to modify the inflammatory response.

From the preceding it is apparent that enhancing inhibitory receptor expression should protect the organism from IC-mediated inflammation. This prediction has been realized in the observation that the anti-inflammatory activity of IVIG results from its ability to upregulate expression of Fc $\gamma$ RIIB, thereby raising the threshold required for ICs to trigger Fc $\gamma$ RIII activation (18). The cytokine responsible for this upregulation has not been identified, but it is likely to fall within the class of anti-inflam- matory cytokines. These reciprocal effects on $\mathrm{Fc} \gamma \mathrm{R}$ activation and antibody-triggered inflammation are summarized in Figure 2.

Our understanding of the molecular mechanisms that underlie the pathophysiology of IC-triggered diseases has focused attention on the central role of the IgG Fc receptors and the secondary role of complement in these in vivo reactions. Fc $\gamma$ Rs exert their function through the paired expression of activation and inhibition receptors. The ratio of these opposing receptors is therefore the critical factor in determining whether an antibody response will result in inflammatory disease or quiescence. The identification of this critical target for antibody-mediated effects in vivo permits the identification of pathways that modulate these cellular targets. Thus, the factors that regulate this ratio will be central to defining the homeostatic mechanisms that function to direct the inflammatory response to protect the host and prevent inappropriate activation and unbridled inflammatory states. This will be a fertile area for future investigations.

\section{Acknowledgments}

I am grateful to members of my laboratory, in particular Pierre Bruhns and Mikael Karlsson, for their critical comments.

1. Shushakova, N., et al. 2002. C5a anaphylatoxin is a major regulator of activating versus inhibitory Fc $\gamma$ Rs in immune complex-induced lung disease. J. Clin. Invest. 110:1823-1830. doi:10.1172/JCI200216577.

2. Arthus, M. 1903. Injections repetees de serum de cheval cuez le lapin. Seances et Memoire de la Societe de Biologie. 55:817-825.

3. Kohl, J., and Gessner, J.E. 1999. On the role of complement and Fc $\gamma$ receptors in the Arthus reaction. Mol. Immunol. 36:893-903.

4. Ravetch, J.V., and Bolland, S. 2001. IgG Fc receptors. Annu. Rev. Immunol. 19:275-290.

5. Ravetch, J.V., et al. 1986. Structural heterogeneity and functional domains of murine immunoglobulin G Fc receptors. Science. 234:718-725.

6. Daëron, M. 1997. Fc receptor biology. Annu. Rev. Immunol. 15:203-234.

7. Clynes, R., et al. 1999. Modulation of immune complex-induced inflammation in vivo by the coordinate expression of activation and inhibitory Fc receptors. J. Exp. Med. 189:179-185.

8. Ravetch, J.V., and Clynes, R.A. 1998. Divergent roles for $\mathrm{FC}$ receptors and complement in vivo. Annu. Rev. Immunol. 16:421-432.

9. Takai, T. 2002. Roles of Fc receptors in autoimmunity. Nat. Rev. Immunol. 2:580-592.

10. Clynes, R.A., et al. 2000. Inhibitory Fc receptors modulate in vivo cytoxicity against tumor targets. Nat. Med. 6:443-446.

11. Sylvestre, D.L., and Ravetch, J.V. 1994. Fc receptors initiate the Arthus reaction: redefining the inflammatory cascade. Science. 265:1095-1098.

12. Sylvestre, D., et al. 1996. Immunoglobulin Gmediated inflammatory responses develop normally in complement-deficient mice. J. Exp. Med. 184:2385-2392.

13. Ji, H., et al. 2002. Arthritis critically dependent on innate immune system players. Immunity. 16:157-168

14. Sylvestre, D.L., and Ravetch, J.V. 1996. A dominant role for mast cell $\mathrm{Fc}$ receptors in the Arthus reaction. Immunity. 5:387-390.

15. Baumann, U., et al. 2001. Distinct tissue site-specific requirements of mast cells and complement components $\mathrm{C} 3 / \mathrm{C} 5$ a receptor in IgG immune complex-induced injury of skin and lung. J. Immunol. 167:1022-1027.

16. Kalergis, A.M., and Ravetch, J.V. 2002. Inducing tumor immunity through the selective engagement of activating Fcgamma receptors on dendritic cells. J. Exp. Med. 195:1653-1659.

17. Weinshank, R.L., Luster, A.D., and Ravetch, J.V. 1988. Function and regulation of a murine macrophage-specific IgG Fc receptor, Fc gamma R-alpha. J. Exp. Med. 167:1909-1925.

18. Samuelsson, A., Towers, T.L., and Ravetch, J.V. 2001. Anti-inflammatory activity of IVIG mediated through the inhibitory Fc receptor. Science. 291:484-486. 